
allemande

50-1| 2018

Regards franco-allemands sur la justice dans la construction européenne | Les élections législatives de 2017 en Allemagne et en Autriche

\title{
La CDU, miroir des mutations d'une société bouleversée
}

Jean-Louis Georget

\section{(QpenEdition \\ Journals}

Édition électronique

URL : https://journals.openedition.org/allemagne/645

DOI : 10.4000/allemagne.645

ISSN : 2605-7913

Éditeur

Société d'études allemandes

Édition imprimée

Date de publication : 30 juin 2018

Pagination : 115-129

ISSN : 0035-0974

Référence électronique

Jean-Louis Georget, "La CDU, miroir des mutations d'une société bouleversée », Revue d'Allemagne et des pays de langue allemande [En ligne], 50-1 | 2018, mis en ligne le 30 juin 2019, consulté le 04 juin 2022. URL : http://journals.openedition.org/allemagne/645; DOI : https://doi.org/10.4000/allemagne. 645 


\section{La CDU, miroir des mutations d'une société bouleversée}

\section{- Jean-Louis Georget *}

\section{Le choc de la soirée électorale de septembre}

Au soir du 24 septembre 2017, la déclaration d'Angela Merkel, suite aux résultats définitifs des élections, a semblé décalée: alors qu'elle analysait le résultat de l'élection comme une victoire ou du moins un succès au bout de douze ans de mandat, les observateurs constataient de manière incrédule que la CDU avait obtenu son plus mauvais résultat depuis 1949. La réaction de la chancelière pouvait dès lors passer pour une bravade ou au contraire une prise de conscience aiguë d'une situation alambiquée à laquelle elle opposait sa crédibilité et la solidité des institutions. Pourtant cette issue était la résultante de changements que les observateurs n'ont pas su interpréter dans un premier temps.

Cet aboutissement présentait toutefois des analogies avec celui de 2009, qui avait occasionné pour la CDU et le SPD une érosion visible, permettant aux petits partis d'obtenir des scores flatteurs. En 2013 au contraire, la CDU avait repris le combat électoral et obtenu 41,5\% des voix. On peut cependant considérer le résultat de 2017 comme l'effet de mouvements souterrains déjà à l'œuvre depuis le début des années 1990, où a commencé l'effritement des classes moyennes en Allemagne qui a généré une réaction contestataire, mélange diffus de ressentiment envers les plus favorisés et d'angoisse face à un ascenseur social en panne. Sans doute les prémices de ce semiéchec n'ont pas été immédiatement lisibles. En effet, la plupart des électeurs se déclaraient satisfaits du travail gouvernemental et des résultats qu'il avait permis d'obtenir dans de nombreux domaines. Mais la certitude presque résignée d'une victoire du parti de la chancelière a sans doute contribué à la dispersion des voix au sein de l'électorat du grand parti populaire qu'est la CDU. Sûrs de la victoire, les électeurs ont pu penser qu'ils ne feraient courir aucun risque à leur formation en manifestant leurs réserves et déceptions avec leur second bulletin. À cela s'ajoute sans doute une bonne

\footnotetext{
* Professeur des universités à Paris 3 Sorbonne Nouvelle et membre du CEREG (EA 4223).
} 
stratégie de l'Alternative pour l'Allemagne (AfD), qui a martelé dans la dernière partie de la campagne ses thèmes populistes de la scission des élites du peuple et de la détérioration de la situation générale sous les coups de boutoir de la mondialisation, dont l’arrivée des réfugiés n'était que la partie émergée de l'iceberg.

\section{Une analyse du vote ${ }^{(1)}$}

Tant en termes de bulletins nominatifs qu'en termes de voix indirectes en faveur d'un parti, la CDU a reculé. Elle a obtenu 32,9 \% des suffrages indirects, indicateur utile de sa représentativité au plan national. Cela est immédiatement perceptible en termes de sièges: le Bundestag, qui comprend 709 députés, en réserve 200 pour le groupe parlementaire de la CDU qui perd 55 sièges et 46 pour celui de la CSU qui perd 10 sièges.

C'est dans la partie occidentale du pays que la CDU a obtenu sans aucune surprise son meilleur score avec $34,3 \%$ des voix, alors qu'elle a récolté un score médiocre dans la partie orientale de l'Allemagne avec $27,7 \%$ des voix. Toutes régions confondues, c'est la CSU qui a obtenu le meilleur résultat avec 38,8 \% des voix en Bavière. La région ayant le plus voté pour la CDU a été la Rhénanie-Palatinat, le Land le plus défavorable au parti conservateur ayant été Berlin. Si la participation électorale a été accrue, la démocratie chrétienne n'en a que peu profité puisque seuls 380000 nouveaux électeurs sont allés vers elle. Les libéraux ont regagné une partie des deux millions d'électeurs qui avaient rallié le parti conservateur en 2013. En outre, 980000 électeurs ont préféré entendre les sirènes de l'Alternative pour l'Allemagne, 90000 ont changé pour la Gauche et 30000 pour les Verts.

Malgré un climat apaisé et attentiste, la CDU a été la formation la plus pénalisée par la marge d'erreur des instituts de sondage, puisque le vote a été de 3,1\% inférieur à ce qui était prévu. La baisse a été régulière dans les derniers moments de la campagne: la démocratie chrétienne disposait d'un potentiel réservoir de vote de 39,5\% au début du mois d'août 2017 , de $38,5 \%$ à la fin du mois d'août et de $36,5 \%$ au début du mois de septembre. Dans le même temps, l'Alternative pour l'Allemagne a commencé à engranger des intentions de vote qui ont augmenté de 7 à $10 \%$, générant un effet de vases communicants.

\section{Une Allemagne scindée}

Le paradoxe est que le climat international anxiogène aurait dû profiter à la stabilité dont était crédité le gouvernement dirigé par Angela Merkel. En effet, le succès inattendu de Donald Trump, la sortie de la Grande-Bretagne de l'Union européenne ou encore les tensions extrêmes avec la Turquie auraient pu s'avérer être autant d'atouts dans le jeu de la chancelière. Pourtant, contre tous les pronostics, la campagne très discrète d'A. Merkel n'a pas porté ses fruits, au contraire des joutes électorales précédentes. En effet, l'électorat a fait preuve contre toute attente d'un regain de goût et d'intérêt pour la politique de sorte que ceux qui ont été les plus bruyants et les plus offensifs dans une campagne atone ont été également les mieux entendus. Si, à la fin

1 Viola Neu, Sabine Pokorny, Bundestagswahl in Deutschland am 24. September 2017. Wahlanalyse, Berlin, Konrad-Adenauer-Stiftung, Hauptabteilung Politik und Beratung, 2017. 
du mois d'août 2017, les questions de la vie quotidienne dominaient encore la campagne, le débat sur les réfugiés a repris de l'ampleur au mois de septembre, passant de 32 à $43 \%$ en termes d'importance dans les sondages, soit le taux le plus élevé depuis février 2016. Ce débat refoulé resurgissait notamment dans les préoccupations des classes sociales les plus fragiles.

À l'instar de ce qui s'est passé dans la majorité des pays occidentaux, une partie de la population allemande a cédé aux arguments du populisme, accréditant la thèse maintenant bien connue d'un glissement à droite de l'ensemble des opinions européennes après trente années de libéralisation et de financiarisation des économies des grands pays occidentaux et d'érosion progressive des classes moyennes sous le double coup de boutoir d'une informatisation accrue des métiers qu'elles exerçaient et d'une dérégulation continue de la législation sociale. Cette ligne de fracture a structuré l'élection, opposant d'une part des groupes sociaux cosmopolites, libéraux et bien intégrés dans la mondialisation et d'autre part des communautés de plus en plus précarisées, pratiquant un repli national et passant de l'abstentionnisme aux partis extrêmes, ce qui explique le glissement d'une partie des électeurs vers l'Alternative pour l'Allemagne, mais également vers la Gauche. En termes de représentativité, cela signifie que la grande coalition rassemble $53 \%$ de l'électorat allemand alors que ce pourcentage s'élevait à 73,5\% en 2013. Mais plus grave, seuls $42 \%$ des électeurs de la classe moyenne se reconnaissent en elle, c'est-à-dire quatre électeurs sur dix provenant de milieux disparates et recouvrant en réalité aujourd'hui des groupes très différents.

\section{La CDU, parti des cosmopolites nantis ${ }^{(2)}$}

En effet, les électeurs de la CDU en 2017 ont le profil le plus équilibré de l'ensemble de l'électorat allemand puisqu'ils sont les bénéficiaires des avancées économiques, culturelles et techniques de l'époque: $52 \%$ d'entre eux plébiscitent la mondialisation, tandis que $48 \%$ restent dubitatifs et angoissés face à la rapidité des mutations qui les entourent. Les classes privilégiées ont voté à $44 \%$ pour la démocratie chrétienne, bien au-delà des 32,9\% de l'adhésion obtenue par la CDU/CSU au plan national. Mais cette victoire n'est que l'envers de la figure de Janus du vote de cette catégorie privilégiée: $6 \%$ des électeurs qui disaient en relever sont revenus vers les libéraux ou ont choisi l'aventure de l'Alternative pour l'Allemagne. On peut néanmoins dire que le milieu intellectuel et libéral au pouvoir d'achat confortable constitue la base de la CDU. Le monde agricole qui lui a été longtemps favorable a quant à lui pratiquement disparu et l'élément confessionnel a reculé au profit d'autres différences liées aux bouleversements du monde de ces 30 dernières années. Il était donc logique que la chancelière se tournât vers les deux partis, le FDP et les Verts, qui correspondaient le mieux à cet électorat favorable à la mondialisation pour essayer de constituer une coalition cohérente, prenant le risque de ne représenter qu'une faible majorité du corps électoral, ouest-allemande et urbaine, et négligeant précisément les groupes sociaux qui lui avaient fait défaut.

2 Thomas Petersen, «Nur scheinbar ruhig», Die politische Meinung, n 456, septembre/octobre 2017. 


\section{La tentative de coalition jamaïcaine}

«La mise en place de nouveautés demande du temps», disait Konrad Adenauer. Lors des discussions préliminaires que la chancelière a menées avec le FDP et les Verts, sans évoquer la gardienne du dogme conservateur qu'a continué d'être la CSU, trois questions ont été abordées, qui constituaient autant de sujets potentiels de conflits : les réfugiés, le réchauffement climatique et l'équation financière de l'Allemagne. Sans doute Angela Merkel a-t-elle pensé qu'elle arriverait à ses fins au terme des discussions préliminaires. La nécessité de former un gouvernement semblait être une priorité pour l'Allemagne et lui offrait la possibilité de redevenir pour la quatrième fois chancelière. Pourtant, au terme de négociations couronnées de succès, les véritables discussions quant à la possibilité de constituer une coalition solide et viable n'avaient pas encore été débattues.

Le 19 novembre, après des débats discrets, les augures semblaient encore favorables. Personne ne désirait de nouvelles élections, les pierres d'achoppement entre les différents partis restant toujours les mêmes: le climat, le charbon, la fiscalité des célibataires et les réfugiés. Cependant, les positions s'étaient rapprochées de sorte qu'une proposition de compromis semblait possible. Sans doute les Verts avaient-ils poussé les négociations jusqu'à la limite de leur capacité d'acceptation. Les chrétiens-démocrates et les écologistes étaient les plus soucieux d'assumer réellement le pouvoir. Le sujet le plus épineux apparaissait être celui du regroupement familial de sorte qu'un accord dans ce domaine pouvait porter les espoirs d'une concorde en matière de climat et de politique fiscale. Les chrétiens-démocrates avaient proposé que le nombre de 200000 migrants par année constitue le cadre de la politique migratoire, ce qui avait été accepté par les écologistes. De la sorte, on pouvait envisager des solutions en matière de rapprochement familial acceptables pour chacun des partenaires, d'autant que la CSU n'avait pas réagi officiellement aux propositions ainsi formulées, accréditant une sortie de crise potentiellement proche. La suite est connue: par ambition personnelle et dans un geste égotique d'irresponsabilité politique, Christian Lindner, le jeune chef du parti libéral, qui avait reconquis un groupe parlementaire au Bundestag, mit fin aux espoirs d'une configuration inédite le 20 novembre au terme d'une déclaration fracassante. Chacun put penser alors que l'ère Merkel était peut-être révolue.

\section{L'échec des discussions préliminaires pour une coalition jamaïcaine et les mutations du système politique allemand ${ }^{(3)}$}

Il y a eu de multiples interprétations sur l'échec de la coalition jamaïcaine et sur les errements des différents participants pour dessiner les traits d'une alliance qui n'avait jamais encore été expérimentée à l'échelon fédéral. Il n'est pas illégitime de se poser la question de savoir si l'on était vraiment si proche d'un accord, si l'occasion d'un renouveau du paysage politique a été gâchée ou si au contraire l'avortement précoce d'un attelage improbable a été une chance pour l'Allemagne. Si les moments où la République fédérale a dû tourner une page de son histoire pour éprouver ses

3 Tilman Steffen, Ferdinand Otтo, «Scheitern in Zeitlupe», Die Zeit, 20 novembre 2017; Clara Pfeffer, "Deutschland ist international deutlich geschwächt», Frankfurter Allgemeine Zeitung, 20 novembre 2017; «Presseecho zum Jamaika-Scheitern », Die Welt, 20 novembre 2017. 
institutions ont été multiples, les efforts accomplis après les élections fédérales de 2017 ont eu une portée particulière. Pendant des décennies, la RFA est restée un havre de stabilité économique et politique de sorte qu'elle a acquis presque malgré elle une dimension de pilier incontournable pour la construction européenne. Cette évidence s'est fissurée et l'on peut considérer que la crise pour la constitution du gouvernement pendant l'hiver 2017/18 n'est pas un accident anodin, mais préfigure sans doute des temps plus tourmentés pour la République de Berlin. Le fait que les relations entre les partis conservateurs et les Verts se soient détendues relève plutôt d'un aspect collatéral des négociations; on peut simplement dire que pendant toutes les discussions, la CDU, la CSU et les écologistes ont appris à mieux se comprendre et à rapprocher leurs positions de sorte que cela pourrait faciliter à l'avenir la formation de coalitions entre la démocratie chrétienne et les écologistes aux plans régional et fédéral. Cela dépendra notamment de la permanence de la ligne pragmatique et réaliste du parti écologiste, adoptée sous la pression des sections régionales du Bade-Wurtemberg et du Schleswig-Holstein.

Mais le caractère historique de la situation se situait ailleurs. Pendant des années, les chrétiens-démocrates et les sociaux-démocrates ont pu s'affronter à égalité pour la conquête du pouvoir de sorte que les options de coalitions était restreintes. La formation des gouvernements s'ensuivait logiquement. Les premières failles dans ce système sont apparues en 2005 lorsque fut formée à contrecœur la seconde grande coalition de l'histoire allemande. Jusqu'alors, le processus de l'agrégation des gouvernements avait fonctionné de manière très prévisible. Depuis le difficile rapprochement de 1961 entre la CDU et le FDP, les rituels des négociations pour former les coalitions s'étaient peu à peu consolidés comme praxis politique. On suivait la formalisation variable du processus de négociation, la rédaction du document de travail et l'annonce des résultats dans des contrats de coalition. S'y ajoutèrent progressivement deux éléments: le premier était une scénarisation croissante à l'attention de l'opinion publique, le second le nombre croissant des acteurs dans des délégations de négociation avec l'intégration des directions de parti et l'assentiment des militants sur fond de différences de culture politique. Lorsque le SPD fut amené en 2013 par ses instances dirigeantes contre l'opinion de sa base à forger une nouvelle grande coalition, ce résultat semblait être encore conforme à une logique politique. En 2017, la complexité des négociations pour la constitution de la coalition jamaïcaine fut étendue et poussée à un point qui ne semblait plus propice à générer de la confiance et à produire du consensus, mais devenait contre-productif. Cela valait tant pour la pléthore des participants que pour le manque de discipline en matière de communication et de présentation des résultats intermédiaires. Cela conduisit au premier véritable échec en matière de formation de gouvernement dans toute l'histoire de la République fédérale.

\section{Une préfiguration du futur ${ }^{(4)}$}

Par-delà l'aspect anecdotique, l'échec des négociations pour former une coalition jamaïcaine inaugure vraisemblablement pour la République fédérale une période d'expérimentation en matière de politique de coalition. L'Allemagne pourrait à

4 Hans Jörg Hennecke, «Eine Zäsur», Die politische Meinung, janvier/février 2018, nº 548. 
l'avenir être plus souvent gouvernée par des coalitions tirées à hue et à dia, qui ne seraient que faiblement efficaces parce qu'elles mettraient en pratique les objectifs des différents partenaires de manière additionnelle et incohérente. De telles coalitions ne verront que difficilement le jour et souffriront de l'incertitude stratégique et de la faiblesse des directions des partis qui y prendront part. La complexité des négociations pour la constitution et la direction de telles coalitions continueront vraisemblablement de s'accroître.

Même si la CDU et le SPD forment une coalition fragile comme c'est le cas depuis le 4 mars, ce modèle de la grande coalition ne sera plus l'issue naturelle des crises gouvernementales. Les signaux de ce désintérêt progressif étaient déjà lisibles en 2017, même si personne n'y a vraiment prêté garde. La CDU, la CSU et le SPD n'avaient rassemblé que $53 \%$ des voix. Il est tout à fait imaginable que leur capacité à former une majorité commune continue de décroître au plan fédéral, comme cela avait déjà été le cas à Berlin et en Saxe-Anhalt. Les partis radicaux et critiques envers le système institutionnel comme l'Alternative pour l'Allemagne ou la Gauche doivent néanmoins renforcer leur potentiel de mobilisation pour provoquer un bouleversement lorsque les partis du centre offrent un angle d'attaque par leur impossibilité à coopérer, la République fédérale de 2018 n'étant pas l'Italie où l'émiettement partisan conduit au chaos. La CDU se voit octroyer le rôle ingrat d'un parti hégémonique pivot qui doit s'entendre avec des partis divergents et profondément ébranlés pour imaginer des gouvernements stables. Si les partis du centre ne devaient plus être en capacité de former des coalitions ou des majorités composées de deux, trois ou quatre partis, il ne resterait comme solutions, outre une réforme électorale qui accentuerait le caractère majoritaire aux élections mais dont les Allemands ne veulent pas au regard de l'histoire, que deux voies étroites allant à l'encontre de l'expérience politique de la République fédérale. Soit il deviendrait acceptable qu'un des partis radicaux puisse se transformer en l'un des éléments respectables d'une coalition à venir, soit l'idée d'un gouvernement minoritaire gagnerait en attractivité. Les deux options ont été testées au plan régional par le SPD : il a aidé en 2014 une personnalité de la Gauche à devenir ministre-président en Thuringe et avait en 2010 formé un gouvernement minoritaire toléré par la Gauche en Rhénanie du Nord-Westphalie. Il va de soi que la Gauche, surtout dans la partie occidentale du pays, n'est pas l'Alternative pour l'Allemagne et qu'il serait plus difficile pour ce dernier parti de trouver des partenaires. L'expérience autrichienne, différente à bien des égards, montre qu'après les vagues d'indignation, l'indifférence peut permettre ce qui semblait improbable.

\section{La revanche des personnalités politiques sur les institutions}

Outre les conséquences précédemment évoquées sur l'échiquier politique, l'échec de la coalition jamaïcaine a ouvert de nouvelles options qui vont au-delà de la routine du jeu habituel des institutions. Le rôle du président dans la formation de la nouvelle coalition s'est avéré plus important pour la première fois et le restera à l'avenir, car, en cas de blocage, c'est à lui de se prononcer pour des gouvernements de minorité ou de nouvelles élections et de faire valoir le cas échéant l'article 81 de la Loi fondamentale relevant des mesures d'exception pour contourner une majorité négative au Bundestag. Il est clair que l'idée d'un gouvernement minoritaire pose de réelles difficultés. Mais si 
des situations se répètent dans lesquelles il n'y a plus de majorité parlementaire capable d'agir, ce cas de figure devrait perdre une partie de son effet repoussoir. Les sceptiques pourraient même arguer du fait que, depuis longtemps, la plupart des gouvernements fédéraux ont été des gouvernements minoritaires parce qu'ils devaient chercher des majorités au Bundesrat. De la même façon la conception de coalitions où les différents partenaires n'agiraient pas de concert dans tous les domaines, mais se concentreraient sur la coordination de sujets centraux et chercheraient par ailleurs sans égard les uns pour les autres des majorités ponctuelles pour des questions politiques en dehors de la discipline de coalition, devrait s'imposer à l'avenir. Le «mariage pour tous » a donné à l'été 2017 un avant-goût de ce que pourraient être de telles relations à l'intérieur d'un gouvernement fédéral.

Si l'idée d'une coalition jamaïcaine ne doit pas être regrettée, il faut accepter que la République fédérale puisse être conduite à l'avenir par des gouvernements dont l'assise politique se révélerait plus faible qu'elle ne l'a été dans la pratique usuelle des institutions jusqu'à maintenant. Les stratégies de réforme au plan intérieur devraient s'avérer plus difficiles et la République fédérale pourrait perdre en influence politique au plan extérieur. Cela pourrait conduire à des compromis qui affaibliraient le principe de subsidiarité et la capacité concurrentielle de l'Allemagne au sein de l'Union européenne. Pour cette raison, les impulsions venant de la chancellerie devraient devenir à l'avenir beaucoup plus importantes qu'aujourd'hui et la personnalité du chancelier déterminante.

\section{Les égarements de la CSU en Bavière ${ }^{(5)}$}

Les résultats électoraux ont également fragilisé la CSU, qui a remporté une victoire à la Pyrrhus par rapport à son statut de parti majoritaire en Bavière depuis 1949, puisqu'elle est passée de 49,5\% en 2013 à 38,8 \% des suffrages en 2017, soit comme il a été dit une perte de 10 sièges au Bundestag. Ceci a eu pour effet de déstabiliser Horst Seehofer, ministre-président et chef du parti chrétien-social. Ce dernier avait adopté une attitude peu amène envers la chancelière, notamment au moment de la crise des réfugiés, qui faillit se terminer par une scission entre les deux formations sœurs. Outre les critiques incessantes à l'égard d'A. Merkel, le ministre-président bavarois avait tel un Tartarin menacé de présenter des candidats chrétiens-sociaux dans toutes les circonscriptions, ce qui, au regard du caractère extrêmement régional du parti, aurait eu un effet tout à fait marginal sur le résultat des élections et se serait transformé en débâcle pour les candidats de la CSU présentés hors des limites de la plus grande région allemande. En vieux routier, $H$. Seehofer ne voulait pas se présenter lui-même comme tête de liste aux élections, autorisant l'investiture du ministre de l'Intérieur Joachim Hermann en 2017 avec un vote de 98,4\% des voix des militants pour conduire la liste des 75 membres du parti.

Le but affiché de la CSU était de reconquérir l'électorat le plus conservateur lors de ces élections. Le résultat obtenu a provoqué une crise sans précédent au sein de la formation bavaroise, le dogme de Franz Josef Strauß selon lequel il ne pouvait y

5 Roman Deininger, Wolfgang Wittl, «Warum Söder und Seehofer sich nicht ausstehen können», Süddeutsche Zeitung, 23 novembre 2017. 
avoir de formation à la droite du parti chrétien-social ayant volé en éclats. Dès lors, le ministre des Finances de H. Seehofer, Markus Söder, a pensé son heure arrivée pour renverser la statue du commandeur et prendre les commandes de la formation bavaroise. Pendant toutes les négociations sur la possibilité d'une coalition jamaïcaine à Berlin, le ministre-président s'est bien gardé d'y associer son rival et a pris une position de plus en plus radicale à son égard, provoquant une réaction réciproque de la part de son concurrent. La ligne de mire pour la CSU est avant tout celle des élections régionales de l'automne 2018. Elles risquent d'être très périlleuses pour le parti bavarois et remettre en cause en cas d'insuccès la relation privilégiée qui existe entre les partis de l'Union. La question du candidat qui mènera le combat à la tête de la CSU est donc cruciale. Or les deux postes que sont celui de ministre-président et celui de chef de parti ont été mis dans la balance après l'échec des élections de septembre. Le ministre-président est élu par les 101 députés du groupe parlementaire régional tandis que le chef du parti est élu par les 1000 délégués réunis en congrès. M. Söder, Franconien protestant, est parvenu à se faire élire comme tête de liste du parti pour devenir potentiellement ministre-président de Bavière au lendemain d'un succès électoral. H. Seehofer a gardé les rênes du parti, mais les candidats à sa succession ont déjà manifesté leurs ambitions, à l'instar de Manfred Weber, responsable du groupe parlementaire conservateur au Parlement européen, qui a proclamé son intérêt pour le parti. Il a justifié son initiative en disant que la CSU avait besoin d'un contrepoids libéral face à Markus Söder. Mais Alexandre Dobrindt, l'ancien ministre des Transports et des Réseaux numériques, dont le bilan à la tête de son ministère est pour le moins parcimonieux, est également sur les rangs.

\section{Le rôle du président dans l'émergence de la grande coalition ${ }^{(6)}$}

Après l'échec des discussions avec les partenaires libéraux et écologistes, le président de la République fédérale Frank-Walter Steinmeier a repris l'initiative et encouragé les grands partis populaires à aller dans la direction d'une nouvelle alliance pour éviter la formation d'un gouvernement minoritaire ou de nouvelles élections dans lesquelles l'Alternative pour l'Allemagne aurait pu avoir la part belle. Ayant été reçus le 30 novembre 2017, soit plus de deux mois après les élections fédérales, les partis chrétien-démocrate et social-démocrate ont décidé de prendre langue pour mesurer la possibilité d'une entente dans le but de permettre à la chancelière d'effectuer un nouveau mandat. La nécessité venait d'une part de l'étranger et notamment de l'Europe, où l'on s'inquiétait que l'Allemagne ne devînt le grand pays malade de l'Europe eu égard à son poids politique et économique. Mais la pression était également interne car les acteurs de l'économie sociale de marché, en premier lieu les syndicats, plaidaient pour la constitution d'un gouvernement stable sous la forme d'une grande coalition pour répondre aux besoins de modernisation de l'Allemagne, notamment dans les domaines de l'énergie, des transports et de l'éducation. Cela seyait à la fois à Angela Merkel, qui souhaitait éviter de nouvelles élections et était placée dans la situation inconfortable de devoir former une coalition, mais également à $\mathrm{H}$. Seehofer, qui était

6 Stephan Haselberger, Kevin P. Hoffmann, Hans Monath, "Steinmeier drängt zu rascher Regierungsbildung», Der Tagesspiegel, 22 janvier 2018. 
sommé de choisir en Bavière entre son poste de ministre-président et son titre de chef de la CSU. Lorsque le SPD s'est prononcé le 15 décembre pour des discussions préalables aux négociations pour former une coalition, les dés étaient en quelque sorte jetés par nécessité rationnelle. Après avoir initié les négociations le 7 janvier, où chacun des partenaires a affirmé le droit de défendre ses propres intérêts, à l'instar du chef de la CSU H. Seehofer qui avait annoncé qu'il continuerait de suivre une ligne dure pour ce qui concernait la question des réfugiés, les jalons pour une nouvelle grande coalition étaient posés dès le 12 janvier, chacun des partenaires ayant fait preuve de bonne volonté. Après quelques craintes lors de discussions qui se prolongeaient aux alentours du 5 février, la chancelière a remporté son pari deux jours plus tard, mettant fin à la crise qui se prolongeait.

\section{Le contrat de coalition ${ }^{(7)}$}

Pour ce qui concerne le marché du travail, le droit du retour d'un temps partiel au temps complet, déjà évoqué en 2013, doit prendre réalité pour les entreprises de plus de 45 employés. Pour celles qui ont entre 45 et 200 employés, ce droit ne sera accordé qu'à un travailleur sur quinze. La cotisation sur l'assurance chômage doit baisser de 0,3 \%. Les partis conservateurs comme les sociaux-démocrates sont d'accord pour ramener les chômeurs de longue durée sur le marché du travail grâce à des contrats soutenus par la puissance publique. Une loi sur l'immigration de cadres doit réguler et orienter leur arrivée. Les contrats de travail à durée déterminée seront limités dans le temps à un an et demi alors que cette durée était de deux ans.

Dans le domaine de l'éducation, la CDU a cédé aux doléances du SPD. Les partis veulent modifier la Loi fondamentale pour que la fédération puisse participer de manière plus active à la construction d'écoles où les élèves ont cours toute la journée, à l'embauche de leur personnel, les parents ayant le droit de réclamer une place pour leurs enfants. La coalition souhaite doter la réforme universitaire d'un milliard et améliorer le montant des bourses universitaires (BAföG). En outre, les partis veulent augmenter les subventions accordées à la recherche, à l'éducation et à la formation pour les porter à hauteur de 6 milliards.

Pour ce qui concerne le numérique, point de revendication de la CSU en particulier, l'Allemagne souhaite se doter de l'Internet rapide sur l'ensemble de son territoire avec 2025 pour date butoir. Tous les contenus doivent être transportés à la même vitesse, la neutralité du réseau restant garantie. Le droit d'avoir accès à un Internet rapide doit être inscrit dans la loi dès 2025. Dans la période de législature à venir, les partis de coalition veulent créer un fonds de 10 milliards d'euros pour mettre en place les autoroutes de l'Internet.

L'Europe est également l'un des points centraux du programme de coalition puisque l'Allemagne a décidé d'encourager les investissements, de créer dans ce but un budget pour l'eurozone et de cesser progressivement d'encourager les politiques budgétaires restrictives. Les partenaires approuvent la mise en place d'une imposition pour les géants de l'Internet comme Google, Apple, Facebook et Amazon. La coalition souhaite également renforcer les droits des travailleurs dans l'espace économique européen et

7 «Der Koalitionsvertrag von Union und SPD», Die Zeit, 7 février 2018. 
concevoir un pacte social pour l'Europe. Elle veut également mettre en valeur le rôle du Parlement européen et permettre aux citoyens de s'exprimer plus amplement dans le débat sur la réforme de l'Union européenne.

Grand cheval de bataille des partis conservateurs, notamment de la CSU, les allocations familiales doivent être augmentées de $25 €$ par enfant et par mois, la première hausse étant prévue pour l'année en cours. Les autres aides familiales doivent être également rééchelonnées, notamment le supplément salarial pour les enfants dans le cas des familles à faibles revenus. En outre, la coalition souhaite introduire des chèques pour les services familiaux et renforcer le droit des enfants dans la Loi fondamentale.

En matière de finances, dont le ministère reviendra pour la première fois au SPD au grand dam des militants chrétiens-démocrates, la contribution solidaire doit disparaître progressivement, cet allègement devant concerner $90 \%$ des contribuables pour l'actuelle mandature. Les augmentations d'impôts doivent être évitées et le budget ne comprendre aucun endettement. Les régions doivent obtenir d'ici 2021 8 milliards d'euros pour couvrir les frais engendrés par l'arrivée des réfugiés. Pour ce qui concerne ces derniers, le nombre des nouveaux arrivants ne doit pas dépasser 220000 personnes par an, requête récurrente des chrétiens-sociaux. Les demandes d'asile doivent à l'avenir s'effectuer dans le cadre d'infrastructures qui intègrent toutes les étapes de la procédure, depuis la prise en charge jusqu'à l'éventuelle reconduction. Le regroupement familial pour les réfugiés disposant d'une protection limitée, comme par exemple celui des Syriens, reste préservé jusqu'au 31 juillet. À partir de cette date, ce regroupement doit être limité à 1000 personnes par mois.

Dans le domaine de le santé, dont les différentes professions soutiennent majoritairement la CDU, une commission nouvellement créée doit examiner la manière dont les honoraires pour les médecins relevant des caisses publiques et privées peuvent être rapprochés. Cette commission doit avoir fait ses propositions pour la fin de l'année 2019. En outre, la médecine rurale doit être mieux soutenue. Les employeurs et les employés doivent à l'avenir se partager les cotisations pour les caisses d'assurancemaladie, la part la plus importante revenant jusqu'à maintenant aux derniers. Enfin, du fait du manque de personnel et des conditions de travail dans les maisons de retraite et les cliniques, les rémunérations doivent être augmentées et des emplois créés.

La coalition veut également renforcer la protection des citoyens. 7500 emplois de police et 2000 dans le domaine de la justice doivent voir le jour dans la fédération et les Länder. La lutte contre le terrorisme doit être organisée semblablement au plan fédéral. Dans ce domaine, les citoyens possédant la double nationalité peuvent être privés de leurs droits s'ils ont combattu pour une organisation extrémiste à l'étranger.

Pour l'aménagement du territoire, les régions les plus faibles doivent recevoir des aides supplémentaires afin de renforcer l'égalité des chances dans l'ensemble des Länder allemands.

En outre, les partis de coalition ont annoncé vouloir mettre en place un soutien à la culture au plan fédéral. Ils veulent encourager la gratuité dans les établissements relevant de la fédération et promouvoir l'égalité des sexes dans le domaine de la culture et de l'art, en systématisant par exemple la parité dans les jurys. La Deutsche Welle, voix allemande vers l'étranger, doit être dotée de nouvelles subventions pour rattraper ses rivaux européens. 
Les partis conservateurs ont également favorisé leur électorat traditionnel des retraités. Le ratio de la retraite par rapport au salaire perçu antérieurement ne doit pas tomber en dessous de $48 \%$ et la cotisation ne pas dépasser $20 \%$ d'ici 2025 . Les mères de trois enfants ou plus seront favorisées puisqu'elles obtiendront des trimestres pour une troisième année. La CSU a exigé cette clause qui se chiffre à 3,4 milliards d'euros par an.

L'environnement est la portion congrue du programme. La grande coalition a plutôt réduit ses objectifs car les résultats en termes de protection climatique doivent être atteints en 2030, les énergies renouvelables constituant $65 \%$ de la consommation à cette même date. La limitation des voitures polluantes a aussi été évoquée et l'encouragement à l'utilisation de véhicules électriques souligné. Dans ce but, les subventions au transport ferroviaire doivent être maintenues et augmentées. Enfin, l'utilisation du glyphosate doit être supprimée le plus rapidement possible.

Le dernier grand domaine d'entente de la coalition est la politique du logement pour lequel les grands partis veulent dépenser plusieurs milliards, dont deux pour le logement social et deux pour des allègements fiscaux. En outre, les deux grands partis se sont entendus pour renforcer le contrôle sur la hausse des loyers. Une réforme des impôts locaux est également annoncée, le gouvernement fédéral envisageant de faire payer aux communes des taxes plus élevées sur les terrains non construits.

\section{Le parti en ordre de marche(8)}

La réorganisation de la CDU et par conséquent l'après-Merkel a bien commencé. Peter Tauber a démissionné le 26 février de son poste de secrétaire général du parti pour des raisons de santé, mais a été également la première victime du renouvellement tant espéré de la CDU suite aux atermoiements des six derniers mois. Pendant longtemps, P. Tauber avait été une épine dans le pied des caciques conservateurs du parti. Il incarnait encore plus que la chancelière l'orientation prise par la formation chrétienne-démocrate ces dernières années et son retrait marque une césure. En effet, en tant que fidèle soldat, il avait défendu le cours politique quavait initié Angela Merkel: si les chrétiens-démocrates avaient glissé peu à peu vers une ligne plus sociale et libérale, c'était aussi une volonté de la direction de la formation. P. Tauber a soutenu avec vigueur la politique de la chancelière, notamment au moment de la question de l'entrée des réfugiés en Allemagne, de la loi sur l'immigration et du mariage pour tous, suscitant l'ire de l'aile traditionnelle du parti.

L'appel au renouvellement du personnel dans la CDU est aussi un appel à un passage de témoin entre générations. La question de savoir si la CDU était allée trop loin dans sa ligne libérale a été discutée âprement au début du mois de février. Deux ministresprésidents, Armin Laschet de Rhénanie du Nord-Westphalie et Daniel Günther du Schleswig-Holstein, se sont prononcés contre un coup de gouvernail à droite. Le promoteur de cette initiative, Jens Spahn, ancien secrétaire d'État aux Finances et figure de proue des conservateurs du parti chrétien-démocrate, s'est senti naturellement

8 Oliver GeORgI, «Sie zu unterschätzen wäre ein Fehler », Frankfurter Allgemeine Zeitung, 26 février 2018; «98,9 Prozent: Kramp-Karrenbauer ist neue Generalsekretärin», Der Spiegel, 26 février 2018; Nico FrIED, «Kramp-Karrenbauer - die Abräumerin», Süddeutsche Zeitung, 28 février 2018. 
concerné. Au bout de 12 ans de mandat de la chancelière et de 18 ans de mandat de la présidente Angela Merkel, la CDU a été profondément remaniée. Lorsqu'elle avait été choisie comme secrétaire générale le 10 avril 2000, A. Merkel avait exigé de son parti qu'il s'ouvre. Elle n'a pas manqué à sa parole, si bien qu'au terme de son mandat, c'est plutôt le manque de conservatisme de son parti qui est vilipendé. Il faut dire que le profil de la chancelière au moment de sa nomination était le contraire de l'élite masculine ouest-allemande, puisqu'elle était une femme de l'Est protestante. Dans l'affaire des dons, le personnel politique ouest-allemand avait épuisé tout son crédit moral et rendu inévitable son avènement.

Si Angela Merkel a compris que sa succession était déjà en jeu, elle ne veut pas laisser le champ complètement libre à ses adversaires, comme le prouve le choix d'Annegret Kramp-Karrenbauer à la tête des chrétiens-démocrates. C'est un changement notable que personne n'avait escompté. La chancelière l'a d'ailleurs annoncé la veille de sa décision aux cercles dirigeants du parti, au vice-président et au responsable du groupe parlementaire. La surprise provoquée dans la bibliothèque de la fondation Konrad Adenauer n'en a été que plus grande. A. Kramp-Karrenbauer a abandonné son mandat de ministre-présidente de la Sarre pour devenir secrétaire générale de la CDU, ce qui est une nouveauté inédite dans l'histoire du parti. Elle a été choisie pour redonner une voix et un visage aux chrétiens-démocrates et réparer les fissures de la démocratie chrétienne avant qu'elles ne se transforment en fractures.

En effet, la chancelière, à qui on a reproché son manque de courage en ne prenant pas de décision marquante lors de ses premiers mandats, a changé de dimension en acceptant pendant l'été 2015 que les réfugiés puissent venir en Allemagne. Dans le même temps, cette politique généreuse a généré l'émergence de l'Alternative pour l'Allemagne, dont l'irruption brutale dans le paysage politique est la conséquence des décisions prises, trop peu expliquées pour que les parties les plus fragilisées de la population allemande puissent la comprendre. En choisissant la ministre-présidente sarroise, la chancelière espère préserver cette partie de son héritage en combattant ses effets les plus néfastes.

En effet, il s'agit bien maintenant, même si A. Merkel reste au pouvoir quatre années de plus, d'envisager l'avenir. On sait que ces transitions sont délicates, comme le montre l'impossibilité pour un chancelier aussi respecté que Konrad Adenauer d'empêcher Ludwig Erhard de se présenter comme son dauphin ou encore l'impuissance de Helmut Kohl face à l'ambition d'Angela Merkel, lui qui présentait Wolfgang Schäuble comme son successeur potentiel.

\section{Une femme de caractère pour la succession}

Le choix de la chancelière est d'autant plus surprenant qu'A. Kramp-Karrenbauer devait entrer dans un cabinet de grande coalition en tant que ministre de l'Intérieur, fait acté après une longue discussion avec H. Seehofer qui avait accepté de laisser le terrain libre. Pourtant, avant le terme des négociations pour une coalition, la Sarroise avait indiqué à la chancelière son intérêt pour prendre les rênes du parti. Sous l'égide d'A. Merkel, il y avait eu de nombreux secrétaires généraux aux caractères très différents: Volker Kauder très pragmatique, Ronald Pofalla qui aimait les envolées parfois absconses, Hermann Gröhe caractérisé par sa fidélité. Ce qui les unissait était une 
loyauté inconditionnelle envers Angela Merkel. Aucun d'entre eux n'avait émis l'intention de développer des idées personnelles allant à l'encontre du travail gouvernemental conduit par la chancelière. Dans les rares cas où il y avait pu avoir un doute, le cabinet de la chancelière avait fait en sorte de ramener très vite les récalcitrants au bercail.

Le modèle d'A. Kramp-Karrenbauer est bien plutôt l'ancien secrétaire général de Helmut Kohl, Heiner Geissler, qui se décrivait lui-même comme secrétaire exécutif du parti. La suite est connue, puisque Helmut Kohl avait mis fin aux fonctions de son secrétaire général en 1989 lorsqu'il avait eu vent d'une fronde pour le renverser. Sans doute la nouvelle secrétaire générale saura-t-elle rendre de l'âme et de la force à son parti, d'autant plus que les adhérents lui savent gré d'avoir renoncé à la rémunération que lui offrait son poste de ministre-présidente pour prendre le risque d'un pari incertain dans des circonstances difficiles en suivant son instinct politique. Elle a prouvé par le passé qu'elle avait cette capacité. En 2012, elle a mis un terme à la coalition jamaïcaine en Sarre, jugeant que les libéraux n'étaient pas capables de gouverner. Malgré l'opposition d'A. Merkel, la ministre-présidente sarroise a tenu bon. De la même manière, pendant la campagne régionale de 2017, le SPD l'ayant rattrapé dans les sondages, elle a déclaré qu'elle quitterait la politique en cas d'échec, de sorte que la CDU put surmonter son handicap et obtenir plus de $40 \%$ des voix. Ainsi, en gagnant en Sarre, elle a à la fois provoqué la première défaite pour Martin Schulz et revitalisé les chrétiens-démocrates au plan national.

Si A. Kramp-Karrenbauer a déjà été ministre de l'Intérieur, de la Famille, de l'Éducation, de la Promotion des femmes et du Travail, si elle a dirigé plusieurs groupes de travail pendant les négociations pour former une coalition et a surmonté un accident de la route pour être présente lors de la phase finale, elle devra cependant affronter à Berlin les jalousies de ses camarades et combattre les ressentiments des citoyens en colère. Mais le plus grand danger est celui de la proximité avec A. Merkel dont elle diffère par le profil, puisqu'elle a étudié le droit et les sciences politiques, qu'elle est mère de trois enfants et siège au comité central des catholiques allemands. Elle peut cependant compter sur l'effet de surprise de sa nomination et gagner son combat contre l'extrême droite sans faire pencher les chrétiens-démocrates dans un sens qui les conduirait à se replier sur eux-mêmes.

\section{La composition du gouvernement ${ }^{(9)}$}

La chancelière s'est d'ailleurs décidée à aider la nouvelle secrétaire générale à accomplir son dessein comme l'a montré le choix des ministres qu'elle a effectué le 25 février 2018. Tenant compte dans un sursaut de survie politique dont elle est coutumière des critiques qui lui avait été adressées, la chancelière a rajeuni son gouvernement en y faisant entrer quatre jeunes personnalités politiques. Elle a d'abord fait du député Jens Spahn, qui s'était opposé à elle notamment au moment de la crise des réfugiés, son ministre de la Santé. Jusqu'à maintenant, il était secrétaire d'État au ministère des Finances. La responsable de la CDU en Rhénanie-Palatinat, Julia Klöckner, va prendre les rênes du ministère pour l'Agriculture, tenues jusqu'à maintenant par la CSU. Anja Karliczek, députée au Bundestag, incarnera le nouveau visage de l'éducation, elle qui

9 Philipp Wıттвоск, «Merkels Kabinettstück», Der Spiegel, 25 février 2018. 
était jusqu'alors présidente exécutive du groupe parlementaire chrétien-démocrate au Parlement fédéral. Helge Braun doit devenir ministre de la Chancellerie, tandis que Peter Altmaier prendra la tête du ministère de l'Économie. Ursula von der Leyen reste ministre de la Défense alors que Hendrik Hoppenstedt prendra la place de H. Braun au ministère de la Chancellerie et mettra son talent au service des rapports entre la fédération et les régions. Hermann Gröhe, ministre de la Santé, et Johanna Wanka, ministre de l'Éducation, ne feront plus partie du gouvernement. Thomas de Maizière, ministre de l'Intérieur sortant, a critiqué l'extension du périmètre de son ministère au sujet de la construction et de la «petite patrie». C'est H. Seehofer qui le remplacera. Deux autres ministres issus des rangs de la CSU le rejoindront, Andreas Scheuer aux Transports et Gerd Müller au Développement.

\section{La ratification par le parti ${ }^{(10)}$}

Le 26 février, la CDU a ratifié l'accord de coalition entre les partis de l'Union et le SPD et accordé ainsi le feu vert pour une nouvelle grande coalition. Seuls 27 délégués sur 975 se sont prononcés contre l'accord négocié. La chancelière avait encouragé son parti à aller dans le sens de la formation d'un gouvernement comprenant les deux grandes formations traditionnelles, 5 mois s'étant écoulés depuis les élections. J. Spahn a soutenu la chancelière, qualifiant le nouveau contrat de coalition de base solide. Il a martelé que la CDU devait retrouver la confiance de ceux qui s'en étaient détournés, soulignant qu'il ne souhaitait pas qu'il puisse y avoir un parti à la droite de la CDU. Mais il y a eu également des critiques puisque le président du Conseil économique des chrétiens-démocrates Werner Bahlsen s'est opposé à l'accord proposé. Reprenant une vulgate néolibérale éculée, il a souligné que le contrat de coalition mettait en avant la redistribution et n'apportait aucune réponse aux questions qui se posent à l'Allemagne. En outre, Christian Wagner, l'ancien responsable du groupe parlementaire hessois chrétien-démocrate, a exigé au vu du renforcement progressif de l'AfD une réorientation du parti. Les populistes de droite étant devenus la troisième force politique au Bundestag, la CDU se doit de les ramener dans le giron originel, les mauvais résultats des élections nationales étant la preuve, selon l'homme politique hessois appartenant à l'aile droite du parti, d'une analyse erronée de la situation.

Cependant le discours de la chancelière selon laquelle le parti, qui a accompli de grandes avancées au plan politique lors des mandatures précédentes, doit aussi tirer les conséquences des erreurs qu'il a faites, a recueilli un large assentiment des militants. Cela passe selon elle par le renouvellement de la grande coalition, seule possibilité de gouverner l'Allemagne de façon pérenne, ce qui n'a pas été du goût d'un certain nombre de délégués. Cependant, la discipline partisane a été la plus forte, surtout après le choix judicieux qu'a opéré la chancelière pour la composition de son gouvernement. Autant la présence de J. Spahn au gouvernement que celle d'A. Kramp-Karrenbauer à la tête du parti a été appréciée, comme le montre son élection à la présidence du parti avec presque 99 \% des voix, un record pour la CDU. Sans doute l'avenir des chrétiens-démocrates passera par la médiation de l'une de ces figures, opposées sur de nombreux plans mais

10 Günther Bannas, Jonannes LeithäUser, "Stimme einer neuen Leidenschaft», Frankfurter Allgemeine Zeitung, 26 février 2018. 
se rejoignant dans un profil qui tranche avec celui de l'actuelle chancelière. Le grand défi du mandat à venir et de l'inscription de la chancelière dans l'histoire sera celui de renforcer la solidité des institutions européennes de concert avec le président français Emmanuel Macron, à un moment où les populismes menacent l'édifice qui a conforté la prospérité et la paix sur le vieux continent. Après le vote favorable du SPD pour une nouvelle coalition, les premières déclarations d'A. Merkel vont dans cette direction.

\section{Zusammenfassung}

Die CDU hat im September 2017 ein enttäuschendes Wahlergebnis erzielt. Nach dem Scheitern der Jamaika-Koalition hat sie wie im Jahre 2013 die Bedingungen für eine neue Zusammenarbeit mit der SPD geschaffen. Vor dem Hintergrund einer drohenden AfD stellen die Ereignisse der letzten Monate einen politischen und institutionellen Umbruch dar. Für viele Christdemokraten nähert sich das Ende der Merkel-Ära. Die Kanzlerin hat darauf reagiert und ein neues politisches Personal befördert, das allem Anschein nach ihr Erbe zum großen Teil bewahren wird.

\footnotetext{
Abstract

The CDU scored a disappointing election result in September 2017. After the failure to form a "Jamaica coalition", the CDU laid the ground, as in 2013, for a renewed cooperation with the SPD. Against the backdrop of a rising AfD, the events of recent months represent a major political and institutional shift. For many Christian Democrats, the end of the Merkel era is approaching. The Chancellor responded and promoted a new political staff that would seemingly preserve most of her legacy.
} 